\title{
Forced vibration of delaminated Timoshenko beams under the action of moving oscillatory
}

\section{mass}

\author{
M.H. Kargarnovin*, M.T. Ahmadian and R.A. Jafari-Talookolaei \\ School of Mechanical Engineering, Sharif University of Technology, Tehran, Iran
}

Received 23 November 2011

Revised 14 April 2012

\begin{abstract}
This paper presents the dynamic response of a delaminated composite beam under the action of a moving oscillating mass. In this analysis the Poisson's effect is considered for the first time. Moreover, the effects of rotary inertia and shear deformation are incorporated. In our modeling linear springs are used between delaminated surfaces to simulate the dynamic interaction between sub-beams. To solve the governing differential equations of motion using modal expansion series, eigensolution technique is used to obtain the natural frequencies and their corresponding mode shapes necessary for forced vibration analysis. The obtained results for the free and forced vibrations of beams are verified against reported similar results in the literatures. Moreover, the maximum dynamic response of such beam is compared with an intact beam. The effects of different parameters such as the velocity of oscillating mass, different ply configuration and the delamination length, its depth and spanwise location on the dynamic response of the beam are studied. In addition, the effects of delamination parameters on the oscillator critical speed are investigated. Furthermore, different conditions under which the detachment of moving oscillator from the beam will initiate are investigated.
\end{abstract}

Keywords: Delamination, Timoshenko beam, Poisson's effect, moving oscillator, dynamic response, oscillator separation

\section{Introduction}

Having the properties of high strength-stiffness, lightweight, fatigue resistance etc, advanced composite materials are widely used in various structural designs like aircraft, helicopters, automobiles, marine and submarine vehicles besides other engineering applications. However, composites are very sensitive to the damage induced during their fabrication or service life. One of the commonly encountered types of defects or damages in the laminated composite structures is delamination. It is known that a structure becomes more flexible and its dynamic characteristics will change if any delamination is generated while the composite structure is in the service. Hence, the continuous measurement of the dynamic response of a structure offers a method for detection of any sort of damage such as delamination on the structure.

In the last three decades there has been growing interest in studying the free vibration of delaminated beams. Wang et al. [1] have studied the free vibrations of an isotropic beam with a through-width delamination by using four Euler-Bernoulli beams connected at the delamination boundaries. The coupling effect of the longitudinal and flexural motions in the delaminated layers was considered in their formulations. It was found that for beams with

${ }^{*}$ Corresponding author: M.H. Kargarnovin, School of Mechanical Engineering, Sharif University of Technology, 14588-89694, Tehran, Iran. Tel.: +98 216616 5510; Fax: +98 216600 0021; E-mail: mhkargar@ sharif.edu. 
a short delamination close to the midplane the results for the natural frequencies were close to the experimental results. However, according to this study dramatic interpenetration of the delaminated sub-laminates was seen in the case of off-midplane delaminations that is physically impossible. This is because the delaminated layers were assumed to deform 'freely' without touching each other (known as free mode) and thus have different transverse deformation. To avoid this kind of incompatibility, Mujumdar and Suryanarayan [2] proposed a model based on the assumption that the delaminated layers are constrained to have identical transverse deformations. They called this as the constrained mode in contrast with the free mode proposed by Wang et al. [1]. Similar constrained mode approach was used by Tracy and Pardoen [3] on a simply supported composite beam, Hu and Hwu [4] on a sandwich beam, and Shu and Fan [5] on a bimaterial beam.

Valoor and Chandrashekhara [6] extended a model for thick composites to include the effects of the transverse shear deformation and the rotary inertia. In addition, the Poisson's effect was included due to its significance in the analysis of angle-ply laminated beams. They have used the 'constrained mode' model to represent the free vibrational behavior of the delaminated beam i.e. they have assumed that the sub-laminates in the delamination regions have the identical displacements and rotations. In their analysis, it was assumed that the delamination is at the midplane and the in-plane displacement was ignored. To simulate the 'open' and 'closed' behavior between the delaminated layers in the free vibrational analysis, Luo and Hanagud [7] presented an analytical model based on the Timoshenko beam theory by using the piecewise-linear springs. In their work, the spring stiffness would then be equal to zero (0) for the free mode and infinity $(\infty)$ for the constrained mode. Moreover, the effect of coupling between the longitudinal and bending vibrations has been considered in their analysis because it was shown by Shen and Grady [8] that this coupling has significant effect on the natural frequencies and mode shapes of the delaminated beam. It should be mentioned that Luo and Hanagud have ignored the Poisson's effect in their study. Shu and Della have presented a simple analytical solution for the free vibration of composite beams with two non-overlapping [9] and overlapping [10] delaminations using the free and constrained modes. Their formulations were based on the classical beam theory to estimate the lower bound of the natural frequency if the free mode is considered and upper bound of the natural frequency if the constrained mode is considered. Ostachowicz and Zak [11] have presented a study on the damped vibration of a laminated cantilever beam with a single closing delamination using the finite element method.

On the other hand, the vibration of a structure excited by a moving system has been the subject of numerous investigations for more than a century [12] with reference to machining processes and behavior of railway tracks and bridges. In this class in general, three types of problems are considered in the literatures:

i If the inertia of the subsystem is neglected, the problem reduces to that of vibration of the distributed system subjected to a given external moving force and is called the moving force problem.

ii If one takes into account the inertia of the moving subsystem and assumes infinite coupling stiffness between subsystem and the beam, the moving mass problem is obtained.

iii Whereas if the coupling stiffness is finite, we end up with the moving oscillator problem.

Clearly, the last problem is the most general of the three. The dynamic response of an intact beam due to a moving system has received a good amount of attention in the literatures [12-28], whereas to the authors' best knowledge, dynamic analysis of the delaminated beam under the action of moving oscillator has been studied only in Ref. [29].

A presentation of aspects on car body design for mass transit vehicles and methods which had been evolved for the analysis of non-linear flexible railway vehicles by use of normal modes has been presented by Persson and Holgersson [13]. In the end of the paper, results from an experimental modal analysis have been shown. Esmailzadeh and Ghorashi [14] analyzed the effects of shear deformation, rotary inertia and the load distribution span on the vibration of the Timoshenko beam subjected to a traveling mass. Later on, the dynamic response of an unsymmetric composite laminated orthotropic beam subjected to moving loads has been studied by Kadivar and Mohebpour [15]. In this paper, one-dimensional finite element analysis based on the classical lamination and first order shear deformation theories are considered. Moreover, different beam elements with 16, 20 and 24 degrees of freedom are used. In addition, the dynamic response of the considered beam under the action of a moving load has been compared to those of an isotropic simple beam. The formulation also has been applied to the static and free vibration analysis.

Fryba [16] has presented the analytical solutions for the simple problems of simply supported beams with uniform cross-section under moving load. Chen et al. [17] have investigated the effects of the boundary flexibility on the 
vibration of a continuum with a moving oscillator. In there, the flexibility in the boundaries was modeled by linear and transverse springs. The dynamic response of an elastically supported infinite beam to an oscillatory mass with constant velocity has been studied by Mackertich [18]. The shear deformation and rotary inertia has been considered in this paper. The nonlinear dynamics of longitudinal ground vehicle traction have been investigated in detail by Olson et al. [19]. Kargarnovin and Younesian [20] studied the response of an isotropic Timoshenko beam with uniform cross-section and infinite length supported by a generalized Pasternak-type viscoelastic foundation subjected to an arbitrary-distributed harmonic moving load. The governing equations of the motion were solved using complex Fourier transformation in conjunction with the residue and convolution integral theorems. The dynamic response for a Timoshenko or Bernoulli-Euler beam with various boundary conditions subjected to moving concentrated forces have been analyzed by Lou et al. [21].

Lou [22] has presented some new finite-element formulae overcoming the shortcomings of the conventional ones to calculate the sectional forces at any cross-section of a Bernoulli-Euler beam on continuously viscoelastic foundation subjected to concentrated moving loads. The dynamic behavior of a laminated composite beam (LCB) supported by a generalized Pasternak type viscoelastic foundation subjected to a moving two-degree-of-freedom (DOFs) oscillator with a constant traveling velocity has been studied by Ahmadian et al. [23,24]. Semi-analytical solution using the Ritz method has been considerd in their papers. The bending moment-the beam deflection at the beam center and just below the oscillator position have been obtained at different oscillator's velocity. Finally, the corresponding velocity related to the maximum value of those parameters has been determined. Recently, Asphalt trackbed deflection under moving dynamic load has been solved analytically [25]. The rail has been modeled as an Euler beam. Tie and ballast have been modeled as a discrete supporting system. Asphalt trackbed has been modeled as another Euler beam on Winkler foundation (sub-grade soil). Chen et al. [26] have investigated the dynamic stiffness matrix of an infinite Timoshenko beam on viscoelastic foundation to a harmonic moving. A Timoshenko beam on the Pasternak viscoelastic bed subjected to a moving load has been investigated by mode summations modal analysis method [27]. Sapountzakis and Kampitsis [28] have developed a boundary element method for the geometrically nonlinear response of shear deformable beams of simply or multiply connected constant cross-section, traversed by moving loads, resting on tensionless nonlinear three-parameter viscoelastic foundation, undergoing moderate large deflections under general boundary conditions. The beam is subjected to the combined action of arbitrarily distributed or concentrated transverse moving loading as well as to axial loading.

At year 2011 in our own work [29], the dynamic analysis of a delaminated composite beam under the action of moving oscillatory mass based on the classical beam theory has been investigated. The beam was analyzed as four interconnected sub-beams using the delamination limits as their boundaries. The constrained mode has been adopted in dynamic analysis of two overlayed neighboring sub-beams. The continuity and equilibrium conditions have been imposed between the adjoining sub-beams along the beam's length. The beam response variation due to the delamination with respect to the intact beam has been investigated.

To the authors' best knowledge; there is no even single investigation in the literatures on the dynamic response of the delaminated composite Timoshenko beam due to a moving oscillator in which the first order shear deformation theory is considered. Owing to the high ratio of the in-plane modulus to transverse shear modulus, transverse shear deformation effects are pronounced in the composite laminates. Thus, the main contribution of the present work is to analyze the dynamic behavior of the delaminated thick composite beam under the action of moving oscillator taking into account the Poisson's effect, transverse shear deformation and longitudinal-bending coupling. Moreover, it has to be mentioned that the Poisson's effect plays significant role when considering the analysis of angle-ply laminated beams.

\section{Mathematical formulation}

\subsection{Geometry}

Consider a thick composite beam of length $\ell$ and rectangular cross-section of $b \times h$, having an embedded delamination as shown in Fig. 1 of depth $h_{2}$ located at a distance $\ell_{1}$ from the left end which is taken as the origin of the $x-z$ coordinate system. Furthermore, it is assumed that the beam is under the action of an oscillator moving at a 


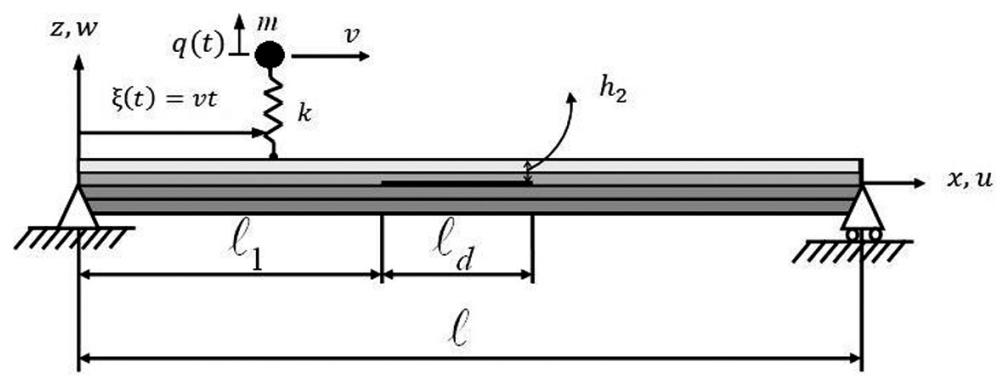

Fig. 1. Delaminated composite beam traversed by a moving oscillator.

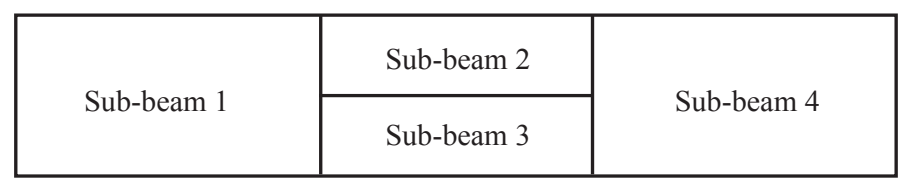

Fig. 2. Representation of beam with delamination into four sub-beams.

constant velocity $v$ along the axial direction of the beam entering from the left end. In order to analyze the dynamic of such beam, Timoshenko beam theory is employed. In addition, $\xi(t)$ denotes the location of the moving oscillator at time $t$. The two parameters $q(t)$ and $k$ are, respectively the vertical displacements of the mass measured from its equilibrium position and oscillator stiffness. When $q=0$, the oscillator is at rest and is subjected to its own weight. Moreover, it should be emphasized that the time interval of $[0, \ell / v]$ will be considered in this analysis.

As it can be seen from Fig. 2, after delamination, the representative beam can be modeled as a combination of four sub-beams connected at the delamination boundaries $x=\ell_{1}$ and $x=\ell_{1}+\ell_{d}$. In this way, we will have four sub-beams of 1 to 4 with lengths and thicknesses of $\ell_{i} \times h_{i}(i=1$ to 4$)$ where $\ell_{2}=\ell_{3}=\ell_{d}, \ell_{4}=\ell-\ell_{1}-\ell_{2}$, $h_{1}=h_{4}=h$ and $h_{2}$ and $h_{3}$ the thicknesses of sub-beams 2 and 3 , respectively. Note that, the reaction force between delaminated sub-beams 2 and 3 is a kind of distributed force modeled by finite number of linear springs [7].

\subsection{Equations of motion}

The governing differential equation for the longitudinal vibration of a delaminated Timoshenko ith sub-beams represented in Fig. 1 is [7]:

$$
\bar{A}_{i} \frac{\partial^{2} u_{i}}{\partial x^{2}}-m_{i} \frac{\partial^{2} u_{i}}{\partial t^{2}}=0, \quad(i=1,2,3 \text { and } 4)
$$

Moreover, the motion equations related to the flexural and transverse vibrations of intact sub-beams 1 and 4 are [7]:

$$
\begin{aligned}
& S_{i} \frac{\partial}{\partial x}\left(\frac{\partial w_{i}}{\partial x}-\psi_{i}\right)-m_{i} \frac{\partial^{2} w_{i}}{\partial t^{2}}=F_{i}(x, t) \\
& \bar{D}_{i} \frac{\partial^{2} \psi_{i}}{\partial x^{2}}+S_{i}\left(\frac{\partial w_{i}}{\partial x}-\psi_{i}\right)-J_{i} \frac{\partial^{2} \psi_{i}}{\partial t^{2}}=\left.0\right|_{(i=1 \text { and } 4)}
\end{aligned}
$$

Similarly for the delaminated sub-beams 2 and 3 are [7]:

$$
\begin{aligned}
& S_{i} \frac{\partial}{\partial x}\left(\frac{\partial w_{i}}{\partial x}-\psi_{i}\right)-m_{i} \frac{\partial^{2} w_{i}}{\partial t^{2}}+q_{i}=F_{i}(x, t) \mid \\
& \bar{D}_{i} \frac{\partial^{2} \psi_{i}}{\partial x^{2}}+S_{i}\left(\frac{\partial w_{i}}{\partial x}-\psi_{i}\right)-J_{i} \frac{\partial^{2} \psi_{i}}{\partial t^{2}}=0
\end{aligned} \mid
$$

in which $F_{3}(x, t)=0$. In these equations, $u_{i}$ and $w_{i}$ denote the longitudinal and flexural displacements, respectively; $\psi_{i}$ is the slope of the deflection curve caused by bending moment. $\bar{D}_{i}$ and $\bar{A}_{i}(i=1$ to 4 ) are modified bending 
stiffness and modified extensional stiffness of either of sub-beams, respectively which are defined as following by including the Poisson's effect [6,30]:

$$
\begin{aligned}
& \bar{A}_{i}=A_{11}-\frac{A_{12}^{2}}{A_{22}}+\frac{\left(A_{12} A_{26}-A_{16} A_{22}\right)^{2}}{A_{22}\left(A_{26}^{2}-A_{22} A_{66}\right)} \\
& \bar{D}_{i}=D_{11}-\frac{D_{12}^{2}}{D_{22}}+\left.\frac{\left(D_{12} D_{26}-D_{16} D_{22}\right)^{2}}{D_{22}\left(D_{26}^{2}-D_{22} D_{66}\right)}\right|_{(i=1,2,3 \text { and } 4)}
\end{aligned}
$$

in which $A_{i j}$ and $D_{i j}$ are given as [30]:

$$
A_{i j}=b \sum_{k=1}^{n_{i}} \bar{Q}_{i j}^{k}\left(z_{k}-z_{k-1}\right), \quad D_{i j}=\frac{b}{3} \sum_{k=1}^{n_{i}} \bar{Q}_{i j}^{k}\left(z_{k}^{3}-z_{k-1}^{3}\right),
$$

Furthermore, $S_{i}, m_{i}$ and $J_{i}$ are the cross sectional shear stiffness, the mass per unit length and the cross-sectional mass moment of inertia, respectively [30]:

$$
S_{i}=k_{s} b \sum_{k=1}^{n_{i}} \bar{Q}_{55}^{k}\left(z_{k}-z_{k-1}\right), \quad m_{i}=b \sum_{k=1}^{n_{i}} \rho^{k}\left(z_{k}-z_{k-1}\right), \quad J_{i}=\frac{b}{3} \sum_{k=1}^{n_{i}} \rho^{k}\left(z_{k}^{3}-z_{k-1}^{3}\right)
$$

where $\rho$ is the mass density of the lamina, $z_{k}$ and $z_{k-1}$ are the location of the $k^{t h}$ lamina with respect to the neutral axis of the $i^{t h}$ sub-beam, $n_{i}$ is the number of plies of the beam, $k_{s}$ is the shear correction factor and $\bar{Q}_{i j}^{k}$ are the transformed material constants [30]. The indicated distributed lateral forces in Eq. (3) i.e. $q_{i}(x, t),(i=2,3)$ are defined as [7]:

$$
\begin{aligned}
& q_{2}(x, t)=k_{f}\left[w_{3}(x, t)-w_{2}(x, t)\right] \\
& q_{3}(x, t)=k_{f}\left[w_{2}(x, t)-w_{3}(x, t)\right]
\end{aligned}
$$

in which $k_{f}$ is known as the spring constant. In addition $F_{i}(x, t)$ in Eq. (3) is the applied external force which varies depending on the oscillator position. For example $F(x, t)$ at time interval of $0 \leqslant t \leqslant \ell_{1} / v$ becomes $-\left[m g+k\left(w_{1}(\xi, t)-q(t)\right)\right] \delta(x-v t)$. More specifically the equation of motion of the moving oscillator at different position can easily be obtained as:

$$
m \ddot{q}+k q= \begin{cases}k w_{1}(\xi, t) \delta(x-v t) & t \leqslant \frac{\ell_{1}}{v} \\ k w_{2}(\xi, t) \delta(x-v t) & \frac{\ell_{1}}{v}<t \leqslant \frac{\ell_{1}+\ell_{d}}{v} \\ k w_{4}(\xi, t) \delta(x-v t) & \frac{\ell_{1}+\ell_{d}}{v}<t \leqslant \frac{\ell}{v}\end{cases}
$$

where $\delta$ is the Dirac-delta function.

In this study in order to perform the dynamic analysis we prefer to use the modal expansion technique. To do this, primarily the natural frequencies and mode shapes of the system out of free vibration analysis have to be obtained. This procedure is dealt with in the next section.

\subsection{Free vibration}

In order to calculate natural frequencies and mode shapes, we set the forcing terms i.e., $F_{i}(x, t)$ in Eqs (2) and (3) to zero. Using Eq. (2), the uncoupled flexural equations of motion for the intact sub-beams 1 and 4 are as follows:

$$
\frac{\partial^{4} w_{i}}{\partial x^{4}}-\left(\frac{m_{1}}{S_{1}}+\frac{J_{1}}{\bar{D}_{1}}\right) \frac{\partial^{4} w_{i}}{\partial x^{2} \partial t^{2}}+\frac{m_{1}}{\bar{D}_{1}} \frac{\partial^{2} w_{i}}{\partial t^{2}}+\frac{J_{1} m_{1}}{\bar{D}_{1} S_{1}} \frac{\partial^{4} w_{i}}{\partial t^{4}}=0, \quad(i=1 \text { and } 4)
$$

Similarly, by using Eq. (3), the coupled flexural equations of motion for the delaminated sub-beams of 2 and 3 are: 


$$
\begin{aligned}
\frac{\partial^{4} w_{2}}{\partial x^{4}} & -\left(\frac{m_{2}}{S_{2}}+\frac{J_{2}}{\bar{D}_{2}}\right) \frac{\partial^{4} w_{2}}{\partial x^{2} \partial t^{2}}+\frac{m_{2}}{\bar{D}_{2}} \frac{\partial^{2} w_{2}}{\partial t^{2}}+\frac{J_{2} m_{2}}{\bar{D}_{2} S_{2}} \frac{\partial^{4} w_{2}}{\partial t^{4}} \\
& =\frac{k}{\bar{D}_{2}}\left(w_{3}-w_{2}\right)+\frac{k J_{2}}{\bar{D}_{2} S_{2}}\left(\frac{\partial^{2} w_{3}}{\partial t^{2}}-\frac{\partial^{2} w_{2}}{\partial t^{2}}\right)-\frac{k}{S_{2}}\left(\frac{\partial^{2} w_{3}}{\partial x^{2}}-\frac{\partial^{2} w_{2}}{\partial x^{2}}\right) \\
\frac{\partial^{4} w_{3}}{\partial x^{4}} & -\left(\frac{m_{3}}{S_{3}}+\frac{J_{3}}{\bar{D}_{3}}\right) \frac{\partial^{4} w_{3}}{\partial x^{2} \partial t^{2}}+\frac{m_{3}}{\bar{D}_{3}} \frac{\partial^{2} w_{3}}{\partial t^{2}}+\frac{J_{3} m_{3}}{\bar{D}_{3} S_{3}} \frac{\partial^{4} w_{3}}{\partial t^{4}} \\
& =\frac{k}{\bar{D}_{3}}\left(w_{2}-w_{3}\right)+\frac{k J_{3}}{\bar{D}_{3} S_{3}}\left(\frac{\partial^{2} w_{2}}{\partial t^{2}}-\frac{\partial^{2} w_{3}}{\partial t^{2}}\right)-\frac{k}{S_{3}}\left(\frac{\partial^{2} w_{2}}{\partial x^{2}}-\frac{\partial^{2} w_{3}}{\partial x^{2}}\right)
\end{aligned}
$$

In free vibrations analysis, one can assume [32]:

$$
u_{i}(x, t)=u_{i}(x) e^{j \omega t} \text { and } w_{i}(x, t)=w_{i}(x) e^{j \omega t}
$$

Substituting Eq. (9) in Eqs (1), (7) and (8), one can obtain:

$$
\begin{aligned}
& \frac{d^{2} u_{i}}{d x^{2}}+\gamma_{i}^{2} u_{i}=0, \quad(i=1,2,3 \text { and } 4) \\
& \frac{d^{4} w_{1}}{d x^{4}}+2 b_{1} \frac{d^{2} w_{i}}{d x^{2}}+c_{1} w_{i}=0, \quad(i=1 \text { and } 4) \\
& \frac{d^{4} w_{2}}{d x^{4}}+2 b_{2} \frac{d^{2} w_{2}}{d x^{2}}+c_{2} w_{2}=d_{2}\left(w_{3}-w_{2}\right)+e_{2}\left(\frac{d^{2} w_{3}}{d x^{2}}-\frac{d^{2} w_{2}}{d x^{2}}\right) \\
& \frac{d^{4} w_{3}}{d x^{4}}+2 b_{3} \frac{d^{2} w_{3}}{d x^{2}}+c_{3} w_{3}=d_{3}\left(w_{2}-w_{3}\right)+e_{3}\left(\frac{d^{2} w_{2}}{d x^{2}}-\frac{d^{2} w_{3}}{d x^{2}}\right)
\end{aligned}
$$

in which:

$$
\begin{aligned}
& \gamma_{i}^{2}=\left.\frac{m_{i} \omega^{2}}{\bar{A}_{i}}\right|_{(i=1,2,3 \text { and } 4)},\left.\quad\left[2 b_{i}=\left(\frac{m_{i}}{S_{i}}+\frac{J_{i}}{\bar{D}_{i}}\right) \omega^{2}, \quad c_{i}=\left(\frac{J_{i} \omega^{2}}{S_{i}}-1\right) \frac{m_{i} \omega^{2}}{\bar{D}_{i}}\right]\right|_{(i=1,2 \text { and 3) }} \\
& {\left.\left[d_{i} \frac{k}{\bar{D}_{i}}\left(1-\frac{J_{i} \omega^{2}}{S_{i}}\right), \quad e_{i}=-\frac{k}{S_{i}}\right]\right|_{(i=1 \text { and 3) }}}
\end{aligned}
$$

At this stage it should be noted that for beams with moderate to high slenderness ratio and low order of delamination opening modes, only one work is reported in which the term $e_{i}$ in above relations has been dropped out because of its smallness [7], nevertheless in this study we are going to consider it in our formulation independent of the value of the slenderness ratio.

From Eqs (10-c) and (10-d), we can get the uncoupled differential equation for the sub-beams 2 and 3 as follows:

$$
\frac{d^{8} w_{i}}{d x^{8}}+\widehat{a}_{1} \frac{d^{6} w_{i}}{d x^{6}}+\widehat{a}_{2} \frac{d^{4} w_{i}}{d x^{4}}+\widehat{a}_{3} \frac{d^{2} w_{i}}{d x^{2}}+\widehat{a}_{4} w_{i}=0,(i=2 \text { and } 3)
$$

where:

$$
\begin{aligned}
& \widehat{a}_{1}=2\left(b_{2}+b_{3}\right)+e_{2}+e_{3}, \widehat{a}_{2}=c_{2}+d_{2}+c_{3}+d_{3}-e_{2} e_{3}+\left(2 b_{2}+e_{2}\right)\left(2 b_{3}+e_{3}\right) \\
& \widehat{a}_{3}=\left(2 b_{2}+e_{2}\right)\left(c_{3}+d_{3}\right)+\left(2 b_{3}+e_{3}\right)\left(c_{2}+d_{2}\right)-e_{2} d_{3}-e_{3} d_{2}, \\
& \widehat{a}_{4}=\left(c_{2}+d_{2}\right)\left(c_{3}+d_{3}\right)-d_{2} d_{3}
\end{aligned}
$$

For a cantilever beam (clamped at $x=0$ ) and for a simply supported beam with movable boundary at the right end, the boundary conditions are, $\left.u_{1}\right|_{x=0}=0,\left.\frac{d u_{4}}{d x}\right|_{x=\ell}=0$, and the longitudinal deformations for each sub-beams out of Eq. (10-a) become: 


$$
\begin{aligned}
& u_{1}=C_{1} \sin \left(\gamma_{1} x\right), \\
& u_{2}=C_{2} \sin \left(\gamma_{2} x\right)+C_{3} \cos \left(\gamma_{2} x\right) \\
& u_{3}=C_{4} \sin \left(\gamma_{3} x\right)+C_{5} \cos \left(\gamma_{3} x\right) \\
& u_{4}=C_{6} \cos \left[\gamma_{1}(\ell-x)\right]
\end{aligned}
$$

On the basis of Eq. (10-b), one can get:

$$
\begin{aligned}
& w_{1}=C_{7} \sin \left(\alpha_{1} x\right)+C_{8} \cos \left(\alpha_{1} x\right)+C_{9} \sinh \left(\beta_{1} x\right)+C_{10} \cosh \left(\beta_{1} x\right) \\
& w_{4}=C_{11} \sin \left(\alpha_{1} x\right)+C_{12} \cos \left(\alpha_{1} x\right)+C_{13} \sinh \left(\beta_{1} x\right)+C_{14} \cosh \left(\beta_{1} x\right)
\end{aligned}
$$

in which:

$$
\alpha_{1}^{2}=b_{1}+\sqrt{b_{1}^{2}-c_{1}}, \quad \beta_{1}^{2}=-b_{1}+\sqrt{b_{1}^{2}-c_{1}}
$$

Having on hand the general solution for $w_{1}$ and $w_{4}$, one can easily obtain following expressions for $\psi_{1}$ and $\psi_{4}$ using Eq. (2):

$$
\begin{aligned}
& \psi_{1}(x)=-C_{7} \bar{\alpha}_{1} \cos \left(\alpha_{1} x\right)+C_{8} \bar{\alpha}_{1} \sin \left(\alpha_{1} x\right)+C_{9} \bar{\beta}_{1} \cosh \left(\beta_{1} x\right)+C_{10} \bar{\beta}_{1} \sinh \left(\beta_{1} x\right) \\
& \psi_{4}(x)=-C_{11} \bar{\alpha}_{1} \cos \left(\alpha_{1} x\right)+C_{12} \bar{\alpha}_{1} \sin \left(\alpha_{1} x\right)+C_{13} \bar{\beta}_{1} \cosh \left(\beta_{1} x\right)+C_{14} \bar{\beta}_{1} \sinh \left(\beta_{1} x\right)
\end{aligned}
$$

where:

$$
\bar{\alpha}_{1}=\frac{\frac{m_{1} \omega^{2}}{S_{1}}-\alpha_{1}^{2}}{\alpha_{1}}, \bar{\beta}_{1}=\frac{\frac{m_{1} \omega^{2}}{S_{1}}+\beta_{1}^{2}}{\beta_{1}}
$$

In certain cases where the $k / E_{\text {avg. }}$ is small, it can be proved that the roots of characteristic equation i.e. Eq. (11), yields to four real and four pure imaginary roots [7]. Under such circumstances, if the roots of Eq. (11) are $\pm \alpha_{2}, \pm \alpha_{3}, \pm \beta_{2}$ and $\pm \beta_{3}$ then, the solutions for Eqs (10-c) and (10-d) become:

$$
\begin{aligned}
w_{2}= & C_{15} \sin \left(\alpha_{2} x\right)+C_{16} \cos \left(\alpha_{2} x\right)+C_{17} \sin \left(\alpha_{3} x\right)+C_{18} \cos \left(\alpha_{3} x\right)+ \\
& C_{19} \sinh \left(\beta_{2} x\right)+C_{20} \cosh \left(\beta_{2} x\right)+C_{21} \sinh \left(\beta_{3} x\right)+C_{22} \cosh \left(\beta_{3} x\right) \\
w_{3}= & C_{15} \hat{\alpha}_{2} \sin \left(\alpha_{2} x\right)+C_{16} \hat{\alpha}_{2} \cos \left(\alpha_{2} x\right)+C_{17} \hat{\alpha}_{3} \sin \left(\alpha_{3} x\right)+C_{18} \hat{\alpha}_{3} \cos \left(\alpha_{3} x\right)+ \\
& C_{19} \hat{\beta}_{2} \sinh \left(\beta_{2} x\right)+C_{20} \hat{\beta}_{2} \cosh \left(\beta_{2} x\right)+C_{21} \hat{\beta}_{3} \sinh \left(\beta_{3} x\right)+C_{22} \hat{\beta}_{3} \cosh \left(\beta_{3} x\right)
\end{aligned}
$$

In which $\widehat{\alpha}_{i}, \widehat{\beta}_{i}$ in above relations are some constant coefficients such as:

$$
\left[\widehat{\alpha}_{i}=\frac{c_{2}+d_{2}-\left(2 b_{2}+e_{2}\right) \alpha_{i}^{2}+\alpha_{i}^{4}}{d_{2}-e_{2} \alpha_{i}^{2}}, \widehat{\beta}_{i}=\frac{c_{2}+d_{2}+\left(2 b_{2}+e_{2}\right) \beta_{i}^{2}+\beta_{i}^{4}}{d_{2}+e_{2} \beta_{i}^{2}}\right]_{(i=2,3)}
$$

Having on hand the general solutions for $w_{2}$ and $w_{3}$, one can easily get the general solutions for $\psi_{2}$ and $\psi_{3}$, using Eq. (3). This yields to:

$$
\begin{aligned}
\psi_{2}=- & C_{15} \bar{\alpha}_{2} \cos \left(\alpha_{2} x\right)+C_{16} \bar{\alpha}_{2} \sin \left(\alpha_{2} x\right)-C_{17} \bar{\alpha}_{3} \cos \left(\alpha_{3} x\right)+C_{18} \bar{\alpha}_{3} \sin \left(\alpha_{3} x\right)+ \\
& C_{19} \bar{\beta}_{2} \cosh \left(\beta_{2} x\right)+C_{20} \bar{\beta}_{2} \sinh \left(\beta_{2} x\right)+C_{21} \bar{\beta}_{3} \cosh \left(\beta_{3} x\right)+C_{22} \bar{\beta}_{3} \sinh \left(\beta_{3} x\right) \\
\psi_{3}=- & C_{15} \tilde{\alpha}_{2} \cos \left(\alpha_{2} x\right)+C_{16} \tilde{\alpha}_{2} \sin \left(\alpha_{2} x\right)-C_{17} \tilde{\alpha}_{3} \cos \left(\alpha_{3} x\right)+C_{18} \tilde{\alpha}_{3} \sin \left(\alpha_{3} x\right)+ \\
& C_{19} \tilde{\beta}_{2} \cosh \left(\beta_{2} x\right)+C_{20} \tilde{\beta}_{2} \sinh \left(\beta_{2} x\right)+C_{21} \tilde{\beta}_{3} \cosh \left(\beta_{3} x\right)+C_{22} \tilde{\beta}_{3} \sinh \left(\beta_{3} x\right)
\end{aligned}
$$

in which:

$$
\begin{aligned}
& \bar{\alpha}_{i}=\frac{\frac{m_{2} \omega^{2}}{S_{2}}-\alpha_{i}^{2}+\frac{k}{S_{2}}\left(\hat{\alpha}_{i}-1\right)}{\alpha_{i}}, \quad \bar{\beta}_{i}=\frac{\frac{m_{2} \omega^{2}}{S_{2}}+\beta_{i}^{2}+\frac{k}{S_{2}}\left(\hat{\beta}_{i}-1\right)}{\beta_{i}} \\
& \tilde{\alpha}_{i}=\frac{\frac{m_{3} \omega^{2}}{S_{3}} \hat{\alpha}_{i}-\alpha_{i}^{2} \hat{\alpha}_{i}+\frac{k}{S_{3}}\left(1-\hat{\alpha}_{i}\right)}{\alpha_{i}}, \quad \tilde{\beta}_{i}=\left.\frac{\frac{m_{3} \omega^{2}}{S_{3}} \hat{\beta}_{i}-\beta_{i}^{2} \hat{\beta}_{i}+\frac{k}{S_{3}}\left(1-\hat{\beta}_{i}\right)}{\beta_{i}}\right|_{(i=2 \text { and 3) }}
\end{aligned}
$$


Equations (12)-(15) and (17) give the general solutions for the delaminated Timoshenko beam. Twenty two unknowns, i.e. $C_{i}(i=1,2, \ldots, 22)$ can be determined by imposing the appropriate boundary and compatibility conditions.

a) The boundary conditions for example:

The cantilever beam:

$$
w_{1}(0)=0, \psi_{1}(0)=0, \psi_{4}^{\prime}(\ell)=0 \text { and } w_{4}^{\prime}(\ell)-\psi_{4}(\ell)=0
$$

the simply supported beam:

$$
w_{1}(0)=0, \psi_{1}^{\prime}(0)=0, w_{4}(\ell)=0 \text { and } \psi_{4}^{\prime}(\ell)=0
$$

Indeed, in our analysis the other types of boundary conditions can be considered without any difficulties. In general, the simply supported and cantilever beams are the most used types of boundary conditions which we are going to focus on them in our analysis in the present work.

b) The compatibility conditions:

at $x=\ell_{1}$ :

$$
\begin{aligned}
& w_{1}=w_{2}, w_{1}=w_{3}, \psi_{1}=\psi_{2}, \psi_{1}=\psi_{3}, u_{2}=u_{1}-H_{2} w_{1}^{\prime}, u_{3}=u_{1}+H_{3} w_{1}^{\prime} \\
& \bar{D}_{1} \psi_{1}^{\prime}=\bar{D}_{2} \psi_{2}^{\prime}+\bar{D}_{3} \psi_{3}^{\prime}-\bar{A}_{2} H_{2} u_{2}^{\prime}+\bar{A}_{3} H_{3} u_{3}^{\prime} \\
& S_{1}\left(w_{1}^{\prime}-\psi_{1}\right)=S_{2}\left(w_{2}^{\prime}-\psi_{2}\right)+S_{3}\left(w_{3}^{\prime}-\psi_{3}\right), \quad \bar{A}_{1} u_{1}^{\prime}=\bar{A}_{2} u_{2}^{\prime}+\bar{A}_{3} u_{3}^{\prime}
\end{aligned}
$$

at $x=\ell_{1}+\ell_{d}$ :

$$
\begin{aligned}
& w_{4}=w_{2}, w_{4}=w_{3}, \psi_{4}=\psi_{2}, \psi_{4}=\psi_{3}, u_{2}=u_{4}-H_{2} w_{4}^{\prime}, u_{3}=u_{4}+H_{3} w_{4}^{\prime} \\
& \bar{D}_{1} \psi_{4}^{\prime}=\bar{D}_{2} \psi_{2}^{\prime}+\bar{D}_{3} \psi_{3}^{\prime}-\bar{A}_{2} H_{2} u_{2}^{\prime}+\bar{A}_{3} H_{3} u_{3}^{\prime} \\
& S_{1}\left(w_{4}^{\prime}-\psi_{4}\right)=S_{2}\left(w_{2}^{\prime}-\psi_{2}\right)+S_{3}\left(w_{3}^{\prime}-\psi_{3}\right), \quad \bar{A}_{4} u_{4}^{\prime}=\bar{A}_{2} u_{2}^{\prime}+\bar{A}_{3} u_{3}^{\prime}
\end{aligned}
$$

By imposing above boundary and compatibility conditions in the obtained general solutions, 22 linear homogenous equations in terms of 22 unknown coefficients $C_{i}(i=1, \ldots, 22)$ are obtained out of which $C_{i}^{\prime}$ s can be calculated. The determinant of the coefficient matrix must be zero to have a nontrivial solution for $C_{i}^{\prime} s$ which forms the characteristic equation for frequencies (eigenvalues). For each frequency, the corresponding mode shape is determined by the eigenvector solution of the equations.

\subsection{Dynamic response}

In this study, we are going to use a piecewise-linear spring model with high stiffness constant (constrained mode) to simulate the behavior between the delaminated surfaces.

Using the separation of variables technique and expressing the dynamic responses of the beam i.e. $w_{i}(x, t)$ and $\psi_{i}(x, t)$ in modal co-ordinates; we have:

$$
w_{i}(x, t)=\sum_{j=1}^{n} W_{i j}(x) q_{j}(t), \psi_{i}(x, t)=\sum_{j=1}^{n} \Psi_{i j}(x) p_{j}(t) \quad(i=1,2,3 \text { and } 4)
$$

where $W_{i j}(x)$ and $\Psi_{i j}(x)$ are the assumed vibration modes satisfying the boundary conditions; $q_{j}(t)$ and $p_{j}(t)$ are the generalized co-ordinates and $n$ is the number of assumed modes.

Substituting Eq. (21) in Eqs (2) and (3) and applying the Ritz method, results in:

$$
\begin{aligned}
& \int_{o}^{\ell_{1}}\left[m_{1} \sum_{j=1}^{n} W_{1 j} \ddot{q}_{j}-S_{1} \sum_{j=1}^{n} W_{1 j}^{\prime \prime} q_{j}+S_{1} \sum_{j=1}^{n} \Psi_{1 j}^{\prime} p_{j}\right] W_{1 k} d x+ \\
& \int_{\ell_{1}}^{\ell_{1}+\ell_{d}}\left[m_{2} \sum_{j=1}^{n} W_{2 j} \ddot{q}_{j}-S_{2} \sum_{j=1}^{n} W_{2 j}^{\prime \prime} q_{j}+S_{2} \sum_{j=1}^{n} \Psi_{2 j}^{\prime} p_{j}-k \sum_{j=1}^{n} W_{3 j} q_{j}+k \sum_{j=1}^{n} W_{2 j} q_{j}\right] W_{2 k} d x+
\end{aligned}
$$




$$
\begin{aligned}
& \int_{\ell_{1}}^{\ell_{1}+\ell_{d}}\left[m_{3} \sum_{j=1}^{n} W_{3 j} \ddot{q}_{j}-S_{3} \sum_{j=1}^{n} W_{3 j}^{\prime \prime} q_{j}+S_{3} \sum_{j=1}^{n} \Psi_{3 j}^{\prime} p_{j}-k \sum_{j=1}^{n} W_{2 j} q_{j}+k \sum_{j=1}^{n} W_{3 j} q_{j}\right] W_{3 k} d x+ \\
& \int_{\ell_{1}+\ell_{d}}^{\ell}\left[m_{1} \sum_{j=1}^{n} W_{4 j} \ddot{q}_{j}-S_{1} \sum_{j=1}^{n} W_{4 j}^{\prime \prime} q_{j}+S_{1} \sum_{j=1}^{n} \Psi_{4 j}^{\prime} p_{j}\right] W_{4 k} d x=F_{k}(t) \\
& \int_{o}^{\ell_{1}}\left[J_{1} \sum_{j=1}^{n} \Psi_{1 j} \ddot{p}_{j}-S_{1} \sum_{j=1}^{n} W_{1 j}^{\prime} q_{j}+S_{1} \sum_{j=1}^{n} \Psi_{1 j} p_{j}-\bar{D}_{1} \sum_{j=1}^{n} \Psi_{1 j}^{\prime \prime} p_{j}\right] \Psi_{1 k} d x+ \\
& \int_{\ell_{1}}^{\ell_{1}+\ell_{d}}\left[J_{2} \sum_{j=1}^{n} \Psi_{2 j} \ddot{p}_{j}-S_{2} \sum_{j=1}^{n} W_{2 j}^{\prime} q_{j}+S_{2} \sum_{j=1}^{n} \Psi_{2 j} p_{j}-\bar{D}_{2} \sum_{j=1}^{n} \Psi_{2 j}^{\prime \prime} p_{j}\right] \Psi_{2 k} d x+ \\
& \int_{\ell_{1}}^{\ell_{1}+\ell_{d}}\left[J_{3} \sum_{j=1}^{n} \Psi_{3 j} \ddot{p}_{j}-S_{3} \sum_{j=1}^{n} W_{3 j}^{\prime} q_{j}+S_{3} \sum_{j=1}^{n} \Psi_{3 j} p_{j}-\bar{D}_{3} \sum_{j=1}^{n} \Psi_{3 j}^{\prime \prime} p_{j}\right] \Psi_{3 k} d x+ \\
& \int_{\ell_{1}+\ell_{d}}^{\ell}\left[J_{1} \sum_{j=1}^{n} \Psi_{4 j} \ddot{p}_{j}-S_{1} \sum_{j=1}^{n} W_{4 j}^{\prime} q_{j}+S_{1} \sum_{j=1}^{n} \Psi_{4 j} p_{j}-\bar{D}_{1} \sum_{j=1}^{n} \Psi_{4 j}^{\prime \prime} p_{j}\right] \Psi_{4 k} d x=0, \quad(k=1,2, \ldots, n)
\end{aligned}
$$

in which using the properties of the Dirac-delta function $F_{k}(t)$ can be written as:

$$
F_{k}(t)=-\left\{\begin{array}{l}
{\left[m g+k \sum_{i=1}^{n} W_{1 i} q_{i}(t)-k q(t)\right] W_{1 k}(v t) t \leqslant \frac{\ell_{1}}{v}} \\
{\left[m g+k \sum_{i=1}^{n} W_{2 i} q_{i}(t)-k q(t)\right] W_{2 k}(v t) \frac{\ell_{1}}{v}<t \leqslant \frac{\ell_{1}+\ell_{d}}{v}} \\
{\left[m g+k \sum_{i=1}^{n} W_{4 i} q_{i}(t)-k q(t)\right] W_{4 k}(v t) \frac{\ell_{1}+\ell_{d}}{v}<t \leqslant \frac{\ell}{v}}
\end{array}\right.
$$

or in a more compact form:

$$
\left\{\begin{array}{l}
\sum_{j=1}^{n} m_{k j} \ddot{q}_{j}+\sum_{j=1}^{n} k_{k j}^{q} q_{j}+\sum_{j=1}^{n} c_{k j}^{q} p_{j}=F_{k}(t) \\
\sum_{j=1}^{n} J_{k j} \ddot{p}_{j}+\sum_{j=1}^{n} k_{k j}^{p} p_{j}+\sum_{j=1}^{n} c_{k j}^{p} q_{j}=0
\end{array}, \quad(k=1,2, \ldots, n)\right.
$$

and in the matrix form:

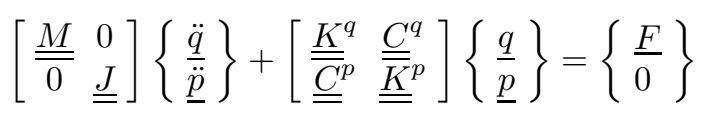

where:

$$
\begin{aligned}
& \underline{\underline{M}}=\left\{m_{k j}, k=1, \ldots n ; j=1, \ldots, n\right\}, \underline{\underline{J}}=\left\{J_{k j}, k=1, \ldots n ; j=1, \ldots, n\right\} \\
& \underline{\underline{K}}^{q}=\left\{k_{k j}^{q}, k=1, \ldots n ; j=1, \ldots, n\right\}, \underline{\underline{K}}^{p}=\left\{k_{k j}^{p}, k=1, \ldots n ; j=1, \ldots, n\right\} \\
& \underline{\underline{C}}^{q}=\left\{c_{k j}^{q}, k=1, \ldots n ; j=1, \ldots, n\right\}, \underline{\underline{C}}^{p}=\left\{c_{k j}^{p}, k=1, \ldots n ; j=1, \ldots, n\right\} \\
& \underline{q}=\left\{q_{1}, q_{2}, \ldots, q_{n}\right\}^{T}, \underline{p}=\left\{p_{1}, p_{2}, \ldots, p_{n}\right\}^{T}, \underline{F}=\left\{F_{1}, F_{2}, \ldots, F_{n}\right\}^{T}
\end{aligned}
$$

It should be mentioned that the two above matrices equations and Eq. (6) are coupled second-order ordinary differential equations. 
Table 1

Fundamental frequencies $(\mathrm{Hz})$ of the beam with single delamination located at interface 3

\begin{tabular}{|c|c|c|c|c|c|c|c|c|c|}
\hline \multirow{2}{*}{$\begin{array}{l}\text { Delamination } \\
\text { Length }(\mathrm{mm})\end{array}$} & \multicolumn{2}{|c|}{ Present } & \multirow{2}{*}{$\begin{array}{l}\text { Ahmadian et al. [29] } \\
\text { Cons. }\end{array}$} & \multicolumn{2}{|c|}{ Della and Shu [9] } & \multicolumn{2}{|c|}{ Luo and Hanagud [7] } & \multicolumn{2}{|c|}{ Shen and Grady [8] } \\
\hline & Cons. $^{\mathrm{a}}$ & Free & & Cons. & Free & Cons. & Free & Averaged test & Cons. \\
\hline Intact & 81.87 & 81.87 & 81.88 & 81.88 & 81.88 & 81.86 & 81.86 & 79.83 & 82.04 \\
\hline 25.4 & 82 & 82 & 81.57 & 81.57 & 81.57 & 82.02 & 82.01 & 80.12 & 81.46 \\
\hline 50.8 & 80.77 & 80.73 & 80.25 & 80.25 & 80.23 & 80.79 & 80.74 & 79.75 & 79.93 \\
\hline 76.2 & 77.8 & 77.51 & 77.27 & 77.27 & 77.16 & 77.82 & 77.52 & 76.96 & 76.71 \\
\hline 101.6 & 73.12 & 71.71 & 72.66 & 72.66 & 72.2 & 73.15 & 71.73 & 72.46 & 71.66 \\
\hline
\end{tabular}

${ }^{\mathrm{a}}$ Cons. stands for the constrained mode.

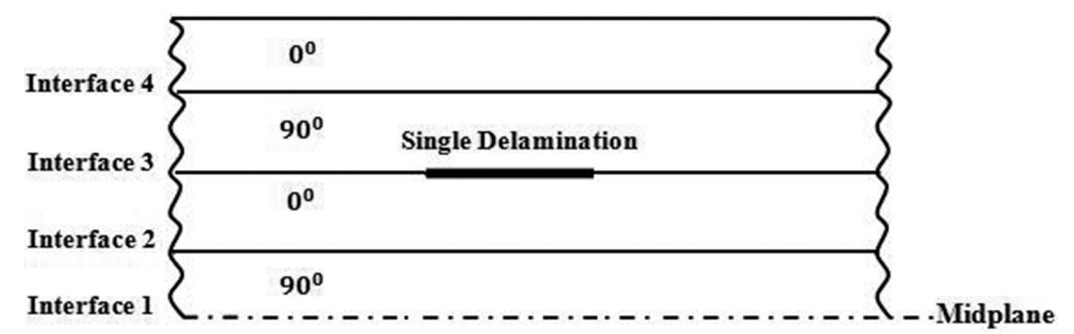

Fig. 3. Interface locations of the delaminations for a $[0 / 90]_{2 s}$ Graphite/Epoxy composite laminate [8].

In order to enter to the calculation phase, numbers of different computer programs using MATLAB Software are developed out of which primarily the natural frequencies and corresponding mode shapes will be obtained. Then, based on the obtained results for the mode shapes, the dynamic response of the delaminated composite beam will be done. In the following section, detailed analysis will be discussed.

\section{Numerical results and discussions}

In this section primarily, different case studies for free vibration analysis will be presented through which the accuracy and efficiency of the present method will be discussed. To check on the accuracy of the presented method in the free vibration analysis, the obtained results are compared with those existing results for a cantilever beam with a single delamination. Then, the dynamic response of a delaminated thick composite beam will be investigated numerically and the effect of different parameters on the dynamic response will be discussed.

\subsection{Free vibration analysis: Cantilever composite beam with a single delamination}

In this case, the delaminated layers i.e., sub-beams 2 and 3, are assumed to vibrate independently (free mode) or vibrate together (constrained mode) one at a time. These vibrational modes can be modeled by setting the spring stiffness to zero $(0)$ or infinity $(\infty)$ for the free and the constrained modes, respectively. In this way, we can estimate the lower and upper bounds for the natural frequency using the free mode and constrained mode, respectively [10].

In order to check on the validity of the obtained results out of the developed computer program primarily, the obtained results for the free vibration analysis of a cantilever composite beam with a single delamination will be compared with those reported by the experimental and analytical analysis [8], with analytical results based on the Timoshenko beam theory [7], with analytical results based on the classical beam theory [9,10,29].

The beam is made of T300/934 graphite/epoxy with a [0/90] $]_{2 s}$ and material properties as used in Ref. [8]. The dimensions of the beam are $127 \times 12.7 \times 1.016 \mathrm{~mm}^{3}$. Different centrally located delaminations are considered one at a time with lengths of 25.4, 50.8, 76.2 and $101.6 \mathrm{~mm}$. The location of this delamination along the thicknesswise direction is shown in Fig. 3; however, this position can be altered to other interfaces with no restriction.

The fundamental frequencies of the free mode and constrained mode considering Poisson's effect are presented in Table 1. It should be mentioned that by ignoring Poisson's effect, the obtained results using presented model become exactly the same as those given in [7], hence for brevity they are not listed in this table. A close inspection on listed results in this table indicates that very good agreement between the frequencies predicted by the present solution and the other reported data in the literatures is seen. 


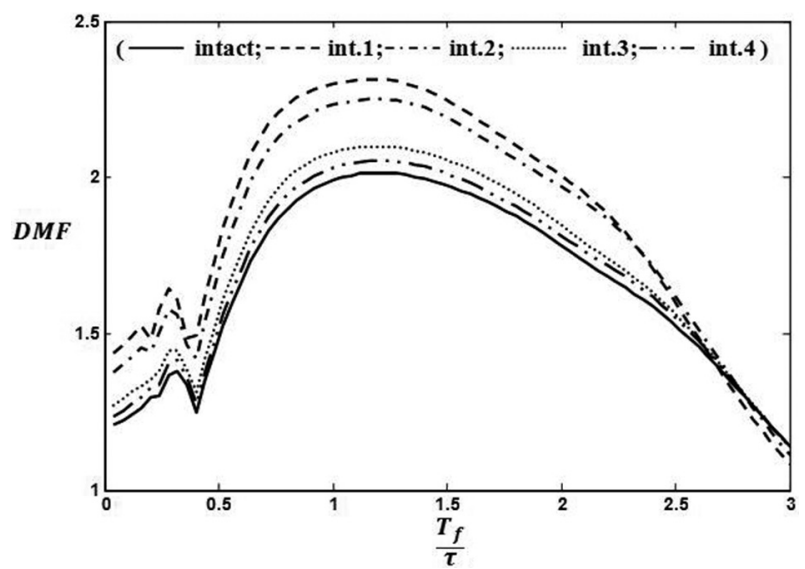

(a)

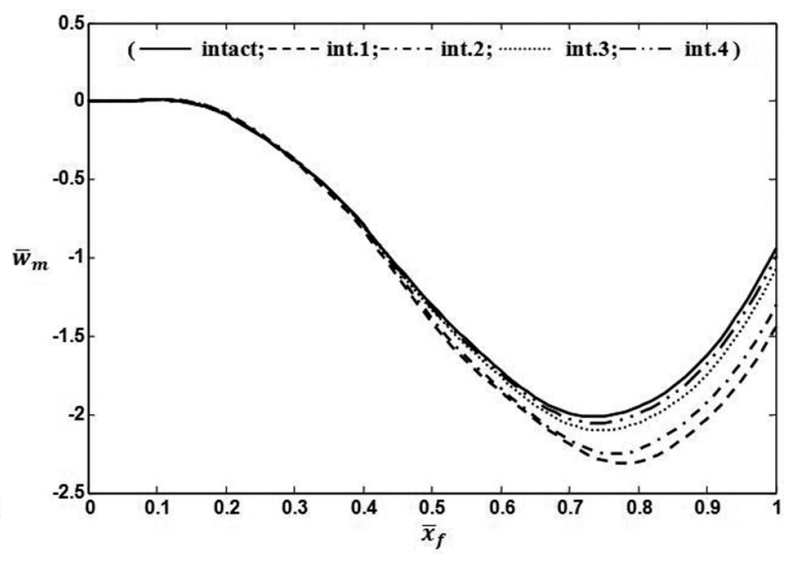

(b)

Fig. 4. a) Variation of DMF vs. $\bar{T}$ for different vertical locations of delamination, b) Variation of $\bar{w}_{m}$ vs. $\bar{x}_{f}$ for different vertical locations of delamination at oscillator critical velocity $\left(\bar{L}_{1}=0.3, \bar{L}_{d}=0.4\right)$.

\subsection{Dynamic response of a composite Timoshenko beam due to a moving oscillator}

Let's define the dynamic magnification factor (DMF) as the ratio of the maximum magnitude of the dynamic deflection at the midpoint of the beam to the corresponding static values of the intact beam. Moreover, let's indicate the $\bar{w}_{m}$ as the dynamic deflection of the beam's midspan at the oscillator critical velocity and $\bar{x}_{f}$ is the non-dimensional time i.e. $\left(\frac{t}{\tau}\right)$ or oscillator non-dimensional horizontal position $\left(\frac{v t}{\ell}\right)$ in which $\tau$ represents the traveling time of the moving oscillator from the left end of the beam to the right end $\left(\tau=\frac{\ell}{v}\right)$ Note that, the critical velocity is the velocity in which the maximum DMF occurs [16].

Based on the self-developed computer programs related to Eqs (6) and (25), following specified geometry, material properties, oscillator parameters and velocity, different results are obtained.

The material properties for the lamina are [23,31]:

$$
\begin{aligned}
& E_{11}=144.8 \mathrm{GPa}, \quad E_{22}=9.65 \mathrm{GPa}, \quad G_{12}=4.14 \mathrm{GPa}, \quad G_{13}=4.14 \mathrm{GPa} \\
& G_{23}=4.14 \mathrm{GPa}, \quad v_{12}=0.3, \quad \rho=1389.23 \mathrm{~kg} / \mathrm{m}^{3}
\end{aligned}
$$

The beam geometries and delamination parameters are:

$$
\ell=10 \mathrm{~m}, b=1 \mathrm{~m}, \mathrm{~h}=0.8 \mathrm{~m}, \ell_{1}=3 \mathrm{~m}, \ell_{d}=4 \mathrm{~m}
$$

Unless mentioned otherwise, the delamination is located at the interface 1 and at the midspan of the beam with the stacking sequence $[0 / 90]_{2 s}$. Also, the magnitude of the oscillating mass, i.e. $m$, is taken to be $0.1 \mathrm{~kg}$. Finally to present results in a standard way, we use the following non-dimensional parameters $\bar{L}_{1}, \bar{L}_{d}$ which represent the delamination spanwise location and length, respectively:

$$
\bar{L}_{1}=\frac{\ell_{1}}{\ell}, \bar{L}_{d}=\frac{\ell_{d}}{\ell}
$$

The following results are presented for a simply supported beam. Nonetheless, similar analyses can be done with no difficulties for other types of boundary conditions. From the static analysis related to the equilibrium condition of a simply supported intact beam, the maximum static deflection under the concentrated mass $m$ is $\frac{m g \ell^{3}}{48 \bar{D}_{1}}$. In the following, in-depth descriptions and discussions will be presented.

\subsubsection{Intact composite beam}

For the intact beam (solid line in Figs 4(a), 5(a) and 6(a)), one could see that DMF increases up to $\bar{T}\left(=\frac{T_{f}}{\tau}\right)=0.3$ and then reaches to a minimum value at $\bar{T}=0.4$. As $\bar{T}$ increases, the maximum value of DMF for the intact beam 
Table 2

Critical velocity $(\mathrm{m} / \mathrm{sec})$ of angle-ply beams with and without considering the Poisson's effect

\begin{tabular}{lccccccc}
\hline$\theta^{\circ}$ & 0 & 15 & 30 & 45 & 60 & 75 & 90 \\
\hline With considering the Poisson's effect & 388.12 & 376.71 & 233.84 & 144.91 & 118.25 & 116.46 & 116.22 \\
Without considering the Poisson's effect & 388.12 & 388.48 & 342.96 & 233.73 & 160.21 & 123.98 & 121 \\
\hline
\end{tabular}

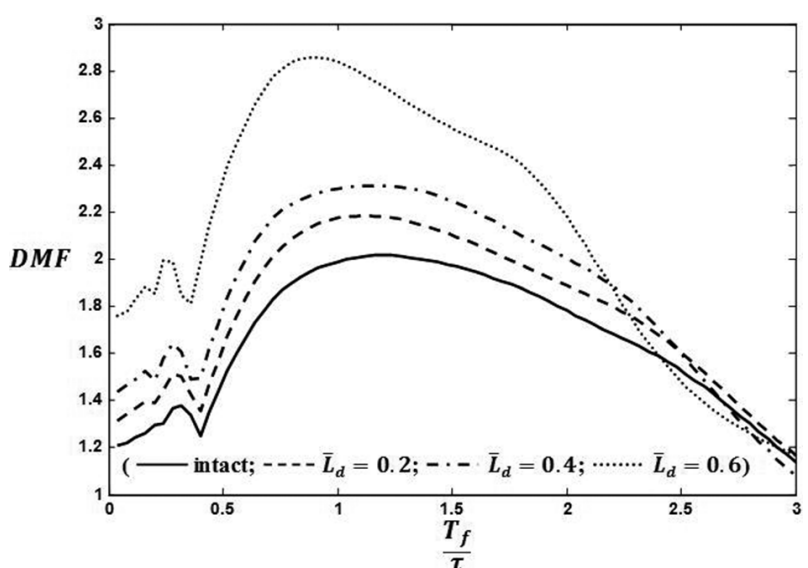

(a)

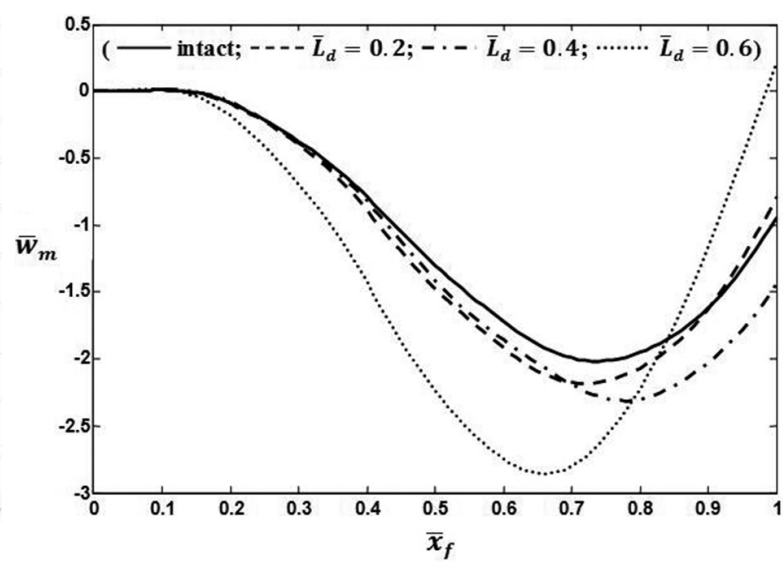

(b)

Fig. 5. a) Variation of DMF vs. $\bar{T}$ for different delamination lengths, b) Variation of $\bar{w}_{m}$ vs. $\bar{x}_{f}$ for different delamination lengths at oscillator critical velocity $\left(\bar{L}_{1}=0.3\right.$, located at interface 1$)$.

occurs at $\bar{T}=1.2$ (corresponding critical velocity for the numerical case; $v_{c}=345.68 \mathrm{~m} / \mathrm{sec}$ ). Note that in these figures, the symbol $T_{f}$ denotes the fundamental period of the intact beam. This type of behavior is also reported in the literature for the moving constant force problem [15,16] and moving oscillatory mass [23,24]. Figures 4(b), 5(b) and 6(b) illustrate the time history of $\bar{w}_{m}$ corresponding to the critical velocity obtained from Figs 4(a), 5(a) and 6(a), respectively. It is shown that the maximum deflection at the beam center occurs when the moving oscillator passes the position of 0.74 of the beam length.

\subsubsection{Delaminated composite beam}

Consider a delamination with length of $\bar{L}_{d}=0.4$ located at the position of $\bar{L}_{1}=0.3$ along the beam length. Figure 4(a) shows the variation of DMF versus $\bar{T}$ for different thicknesswise location of the delamination. For brevity of notations, we denote int. for interface in Fig. 4. The maximum DMF occurs at $\bar{T}=1.2\left(v_{c}=345.68 \mathrm{~m} / \mathrm{sec}\right)$, no matter in which interface the delamination is located (see Fig. 3).

It is shown in Fig. 4(b) that the maximum deflection at the beam center occurs when the moving oscillator passes the position of 0.75 of beam length when the delamination located at the interface 3 or 4 However, this will yield to 0.78 of the beam length when the delamination located at the interface 1 or 2 .

Figure 5 displays the effect of delamination length on the beam response while the delamination position within the beam height is kept unchanged i.e. along the interface 1 at $\bar{L}_{1}=0.3$. Referred to Fig. 5a, it is shown that for the delamination lengths of $\bar{L}_{d}=0.2$ and $0.4\left(v_{c}=322.64 \mathrm{~m} / \mathrm{sec}\right)$ the maximum DMF occurs at $\bar{T}=1.12$ while for the delamination length of $\bar{L}_{d}=0.6\left(v_{c}=253.50 \mathrm{~m} / \mathrm{sec}\right)$, this maximum occurs at $\bar{T}=0.88$. This means that for the delamination length more than half of the beam's length, significant change is seen in the oscillator critical velocity. In addition, it is clearly seen that the existence of any single delamination with length of for instance $\bar{L}_{d}=0.6$ can increase the maximum value of DMF up to $42 \%$.

Figure 5(b) shows the time history of $\bar{w}_{m}$ at the oscillator critical velocity vs. oscillator non-dimensional horizontal position related to the Fig. 5(a). As it is seen from this figure, the maximum deflection at the beam center occurs when the moving oscillator just passes the:

a) 0.72 of the beam length if the delamination length is $\bar{L}_{d}=0.2$,

b) 0.78 of the beam length if the delamination length is $\bar{L}_{d}=0.4$ and 

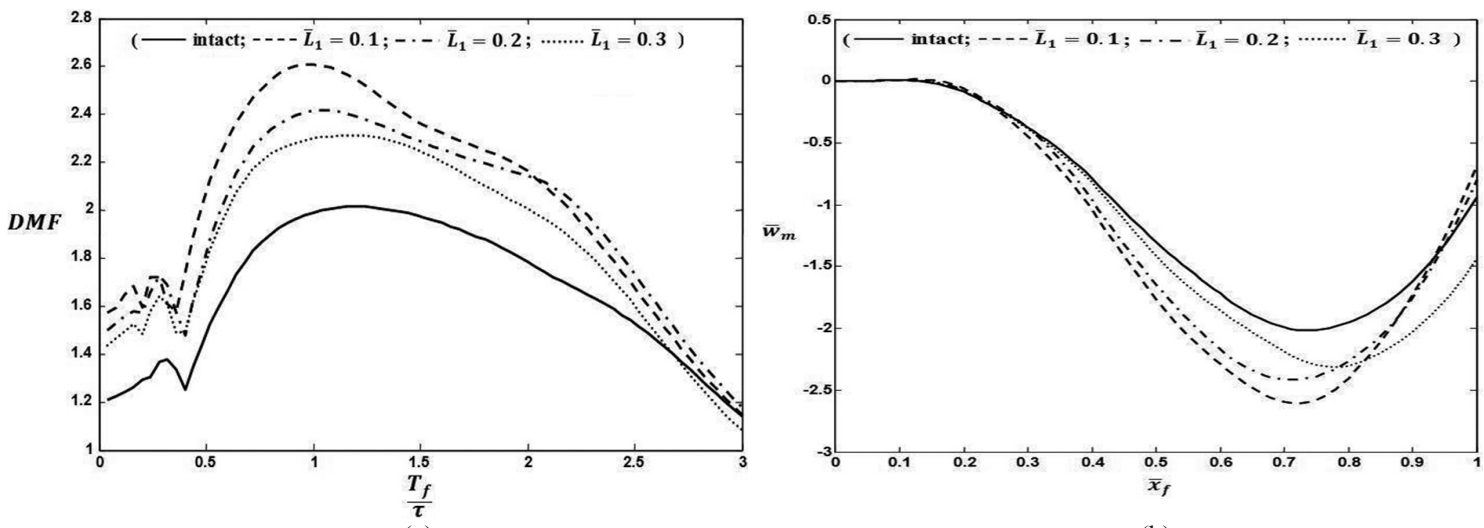

(a)

(b)

Fig. 6. a) Variation of DMF vs. $\bar{T}$ for different delamination spanwise location, b) Variation of $\bar{w}_{m}$ vs. $\bar{x}_{f}$ for different delamination spanwise location at oscillator critical velocity $\left(\bar{L}_{d}=0.4\right.$, located at interface 1$)$.

c) 0.66 of the beam length if the delamination length is increased to $\bar{L}_{d}=0.6$.

Now, consider a delamination with the length of $\bar{L}_{d}=0.4$ along the interface 1 . The influence of delamination spanwise location along this interface on the beam's dynamic response is shown in Fig. 6.

Referred to Fig. 6(a) the maximum DMF occurs at different values of $\bar{T}=1$ and 1.04 for cases where the $\bar{L}_{1}=0.1\left(v_{c}=288.07 \mathrm{~m} / \mathrm{sec}\right)$ and $0.2\left(v_{c}=299.59 \mathrm{~m} / \mathrm{sec}\right)$, respectively, while this maximum occurs at $\bar{T}=1.2$ when $\bar{L}_{1}=0.3\left(v_{c}=345.68 \mathrm{~m} / \mathrm{sec}\right)$. This means that as the delamination gets closer to the left end of the beam, its corresponding critical velocity will decrease accordingly.

As it can be seen from Fig. 6(b) for $\bar{L}_{1}=0.1,0.2$ and 0.3 , the DMF critical speed occurs when the moving oscillator travels $0.72,0.71$ and 0.78 of the beam length, respectively.

As can be seen from Figs 4-6, the dynamic deflection of the delaminated beam (DMF) is larger than the corresponding ones of the intact beam at critical velocity.

The effect of shear deformation on the DMF of the cross-ply beam $\left([0 / 90]_{2 s}\right)$ is shown in Fig. 7. For this case, the length of delamination is $\bar{L}_{d}=0.4$, and it is located along the interface 1 at position of $\bar{L}_{1}=0.3$. It is clear that for small slenderness ratios i.e. $\ell / h$, based on the first shear deformation theory the results tend to deviate from those obtained through the classical lamination theory. But, for large $\ell / h$ ratios, deformation due to shear are negligible as compared to deformations due to flexure and the classical theory suffices to accurately predict the dynamic response of the beam.

The overall observation of Figs 7(a)-(7d) indicates that for the $\ell / h=12.5$ this maximum occurs at $\bar{T}=1.2$ and for $\ell / h$ ratios of the 25,50 and 100 the maximum DMF occurs at the same value of $\bar{T}=1.12$, no matter what kind of theory is used.

The influence of the Poisson's effect on the DMF vs. $\bar{T}$ for the angle-ply beam with stacking sequence of $[\theta /-\theta / \theta /-\theta]_{s}$ is shown in Fig. 8 for two cases i.e. beam by considering and beam by ignoring the Poisson's effect. The angle of orientation is changed from zero $\left(0^{\circ}\right)$ to ninety $\left(90^{\circ}\right)$. As one can see from this figure and can be expected, the Poisson's effect inclusion makes no significant outcome on the DMF for the unidirectional $\left(\theta=0^{\circ}\right)$ or cross-ply $\left(\theta=90^{\circ}\right)$ laminated beams. However, the DMF for an angle-ply beam deviates significantly from the exact value when one ignores the Poisson's effect. The maximum difference occurs at $\left(\theta=45^{\circ}\right)$ in which $65.8 \%$ decrease between the results is seen with respect to the case where the Poisson's effect is included. It should be noted that this huge amount of difference is referred to the time when the travelling force is marching with the critical speed. This phenomenon is also reported in $[6,30]$ for the free vibration analysis. Moreover, the corresponding critical velocities of all abovementioned angle-ply beams are calculated and listed in Table 2 . It can be concluded from Table 2 that the critical velocities decrease as the fiber orientation increases in both cases i.e. considering and ignoring the Poisson's effect.

Figures 9-11 depict the variation of non-dimensionalized contact force $\left(F_{c}=F_{i}(x, t) / m g\right)$ vs. $\bar{x}_{f}$ at different delamination parameters. In these figures, the oscillator parameters are taken as: 


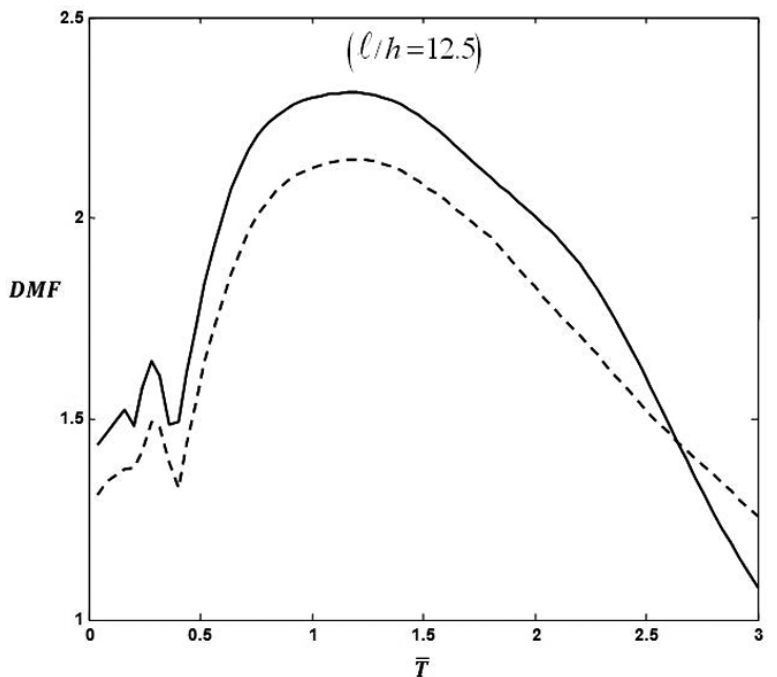

(a)

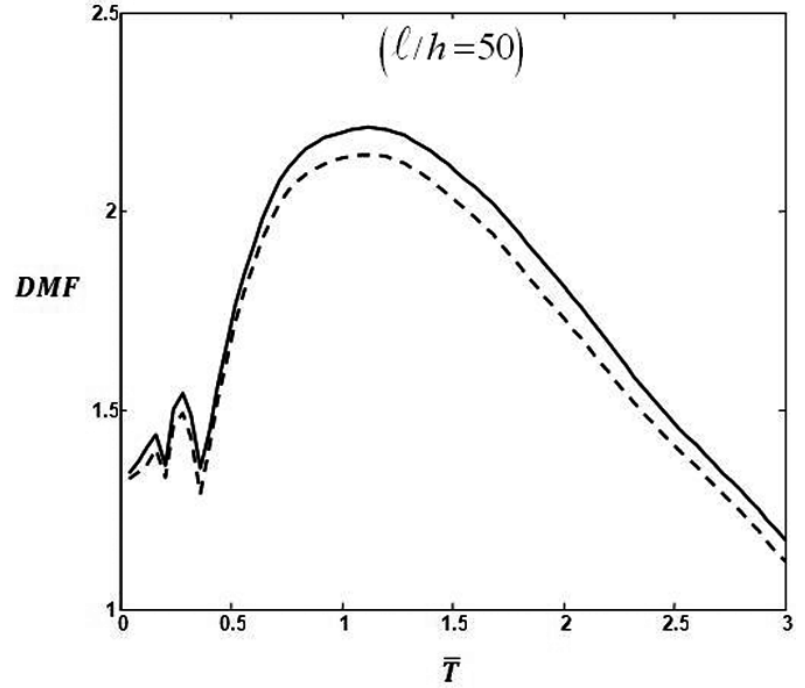

(c)

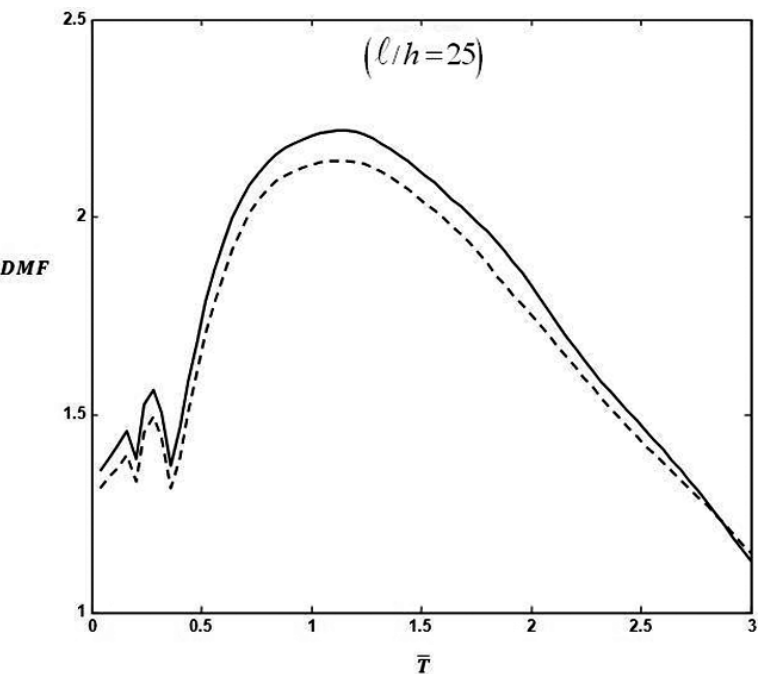

(b)

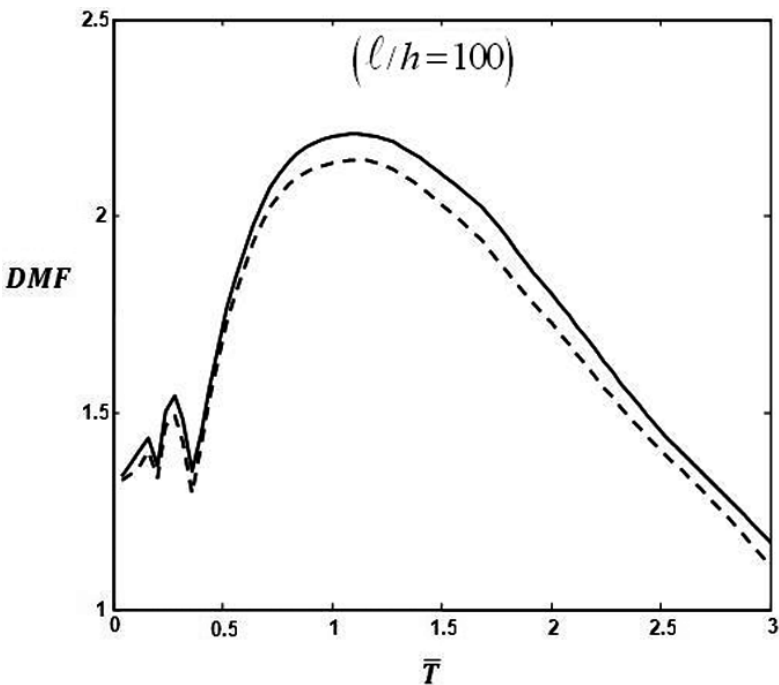

(d)

Fig. 7. Effect of shear deformation on the DMF vs. $\bar{T}$ for different slenderness ratio (— FSDT;

CLT)

$m=0.1 \mathrm{~kg}, v=5 \frac{\mathrm{m}}{\mathrm{sec}}, \mathrm{k}=10^{9} \mathrm{~N} / \mathrm{m}$

It should be mentioned that the separation of the oscillator occurs when the contact force becomes negative. It should be emphasized that when such a separation occurs, the equations of motion are no longer valid to describe the ensuing motion and more complicated analysis needed to be done which is outside of the scope of this study.

Figure 9 displays the effect of delamination thicknesswise location on the normalized contact force. As can be seen, the separation of the oscillator from the beam is found to occur at the value of $\bar{x}_{f}$ approximately equal to 0.796 for the intact beam and at $0.299,0.388,0.407$ and 0.496 when the delamination is located at interface 1, 2, 3 and 4, respectively. It is concluded that as the delamination location shifts towards the mid-plane of the beam, the location of the separation point occurs earlier along the beam length. This type of behavior is similar to the results of the oscillator separation from the delaminated Euler-Bernoulli beam [29].

The influence of delamination length on the normalized contact force is shown in Fig. 10. It is found that, the separation of the oscillator from the beam occurs at the value of $\bar{x}_{f}$ approximately equal to $0.392,0.299$ and 0.284 for delamination length $\bar{L}_{d}$ equal to $0.2,0.4$ and 0.6 , respectively. In this figure it can be seen that as the delamination 

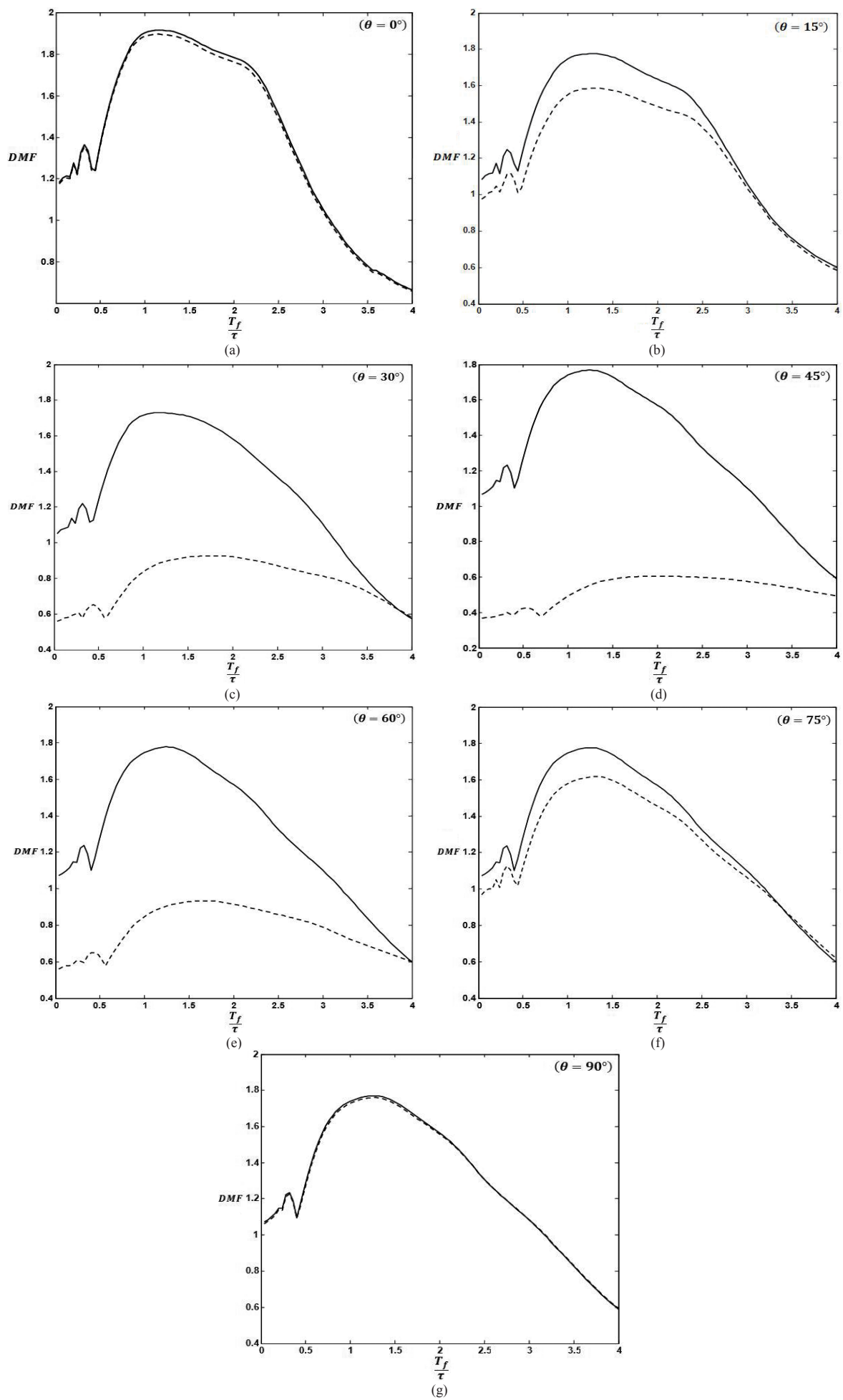

Fig. 8. Influence of the Poisson's effect on the DMF vs. $\bar{T}$ for symmetric angle-ply beam (— With considering the Poisson's effect; - - Without considering the Poisson's effect) 


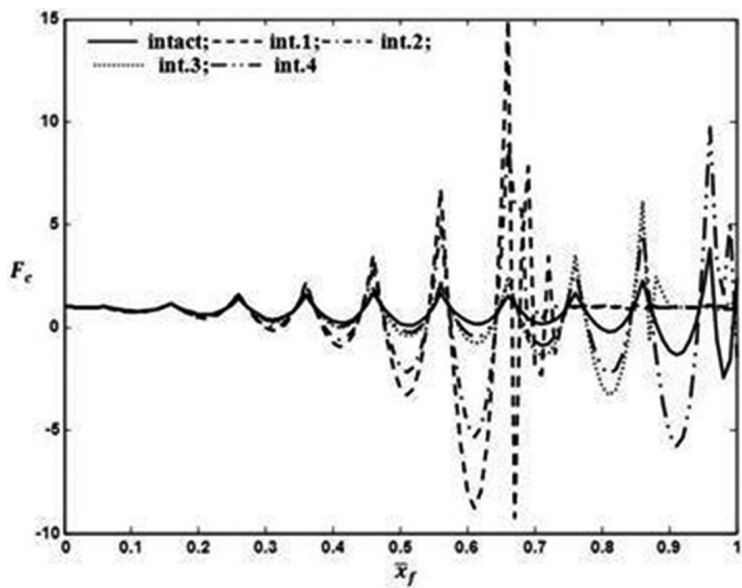

Fig. 9. Effects of thicknesswise location of the delamination on the oscillator separation from the beam $\left(\bar{L}_{1}=0.3, \bar{L}_{d}=0.4\right)$.

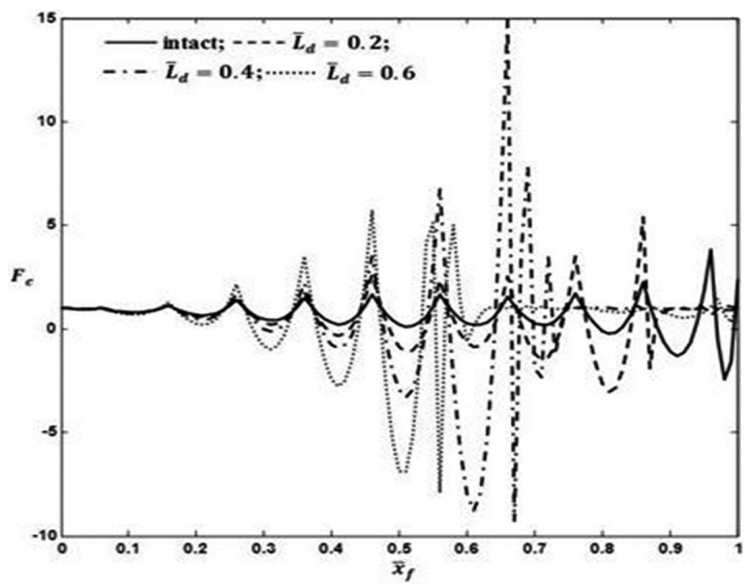

Fig. 10. Effects of delamination length on the oscillator separation from the beam $\left(\bar{L}_{1}=0.3\right.$, interface 1$)$.

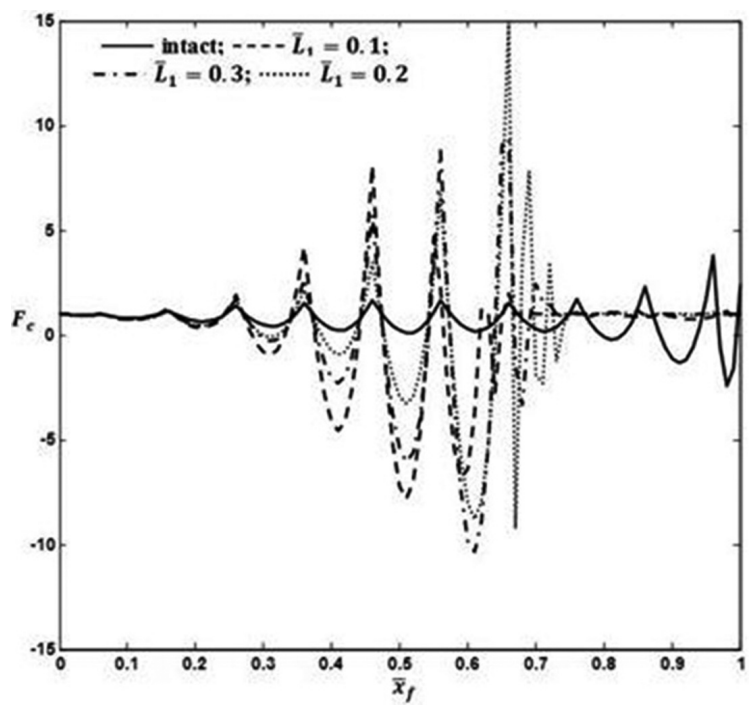

Fig. 11. Effects of spanwise location of the delamination on the oscillator separation from the beam $\left(\bar{L}_{d}=0.4\right.$, interface 1$)$.

length is increased, the oscillator separates earlier along the beam length. Note that the results for the intact beam shown in Figs 10 and 11 are the same as those represented in Fig. 9.

The variation of the normalized contact force versus oscillator position for different spanwise location of the delamination is depicted in Fig. 11. One could see that the oscillator separation takes place at 0.284, 0.294 and 0.299 of the beam length when the $\bar{L}_{1}=0.1,0.2$ and 0.3 , respectively. It can be concluded that the oscillator separation from the delaminated beam occurs earlier as the delamination moves towards the beam boundary.

\section{Conclusions}

The governing differential equations of motion have been derived for a composite Timoshenko beam with a single delamination acted upon by a moving oscillator with a constant speed. By utilizing the modal analysis and Ritz method, we have obtained a set of second order ordinary differential equations Numerical results are presented in 
the present paper for a simply supported beam with a constant oscillator travelling with various prescribed constant speeds. Based on derived results followings are concluded:

1 It is found that the dynamic deflection of the delaminated beam (DMF) is larger than the corresponding ones of the intact beam at critical velocity.

2 For a delaminated beam, when the delamination shifts towards the midplane of the beam, the DMF at the critical velocity occurs as the force position gets closer to the beam's right end.

3 It is found that the dynamic deflection is sensitive to the depth, length and spanwise location of the delamination. The existence of any single delamination and its location can increase the maximum value of DMF for example in some cases up to $42 \%$.

4 For the delamination length more than half of the beam's length, significant change is seen in the force critical velocity.

5 The critical velocity decreases as the delamination gets closer to the beam's left end.

6 The transverse shear deformation effect is pronounced for the thick laminated composite beams.

7 Neglecting the Poisson's effect decreases significantly the DMF for an angle-ply beam.

8 It is shown that the critical velocity has its highest value at ply angle of $0^{\circ}$, and will decrease as the ply angle increases.

9 As the delamination location shifts towards the mid-plane of the beam, the location of the separation point occurs earlier along the beam length.

10 As the delamination length is increased, the oscillator separation from the beam takes place sooner along the beam length.

11 The oscillator separation from the delaminated beam occurs earlier as the delamination moves towards the beam boundary.

\section{References}

[1] J.T.S. Wang, Y.Y. Liu and J.A. Gibby, Vibration of split beams, Journal of Sound and Vibration 84(4) (1982), 491-502.

[2] P.M. Mujumdar and S. Suryanarayan, Flexural vibrations of beams with delaminations, Journal of Sound and Vibration 125(3) (1988), $441-461$.

[3] J.J. Tracy and G.C. Pardoen, Effect of delamination on the natural frequencies of composite laminates, Journal of Composite Materials 23(12) (1989), 1200-1215.

[4] J.S. Hu and C. Hwu, Free vibration of delaminated composite sandwich beams, AIAA Journal 33(7) (1995), 1911-1918.

[5] D. Shu and H. Fan, Free vibration of a bimaterial split beam, Composites: Part B 27(1) (1996), 79-84.

[6] M.T. Valoor and K. Chandrashekhara, A thick composite-beam model for delamination prediction by using neural networks, Composite Science and Technology 60 (2000), 1773-1779.

[7] H. Luo and S. Hanagud, Dynamics of delaminated beams, International Journal of Solids and Structures 37 (2000), 1501-1519.

[8] M.H.H. Shen and J.E. Grady, Free vibrations of delaminated beams, AIAA Journal 30(5) (1992), 1361-1370.

[9] D. Shu and C.N. Della, Free vibration analysis of composite beams with two non-overlapping delaminations, International Journal of Mechanical Sciences 46 (2004), 509-526.

[10] C.N. Della and D. Shu, Free vibration analysis of composite beams with overlapping delaminations, European Journal of Mechanics A/Solids 24 (2005), 491-503.

[11] W. Ostachowicz and A. Zak, Vibration of a laminated beam with a delamination including contact effects, Shock and Vibration 11(3-4) (2004), 157-171.

[12] G.G. Stokes, Discussion of a differential equation relating to the breaking of railway bridges, Transactions of the Cambridge Philosophical Society 8 (1849), 707-735 (reprinted in 1883, Mathematical and Physical Papers, 2, pp. 178-220).

[13] I. Persson and M. Holgersson, Body structure and vehicle dynamics, Vehicle System Dynamics 15 (1986), $441-447$.

[14] E. Esmailzadeh and M. Ghorashi, Vibration analysis of a Timoshenko beam subjected to a traveling mass, Journal of Sound and Vibration 199 (1997), 615-628.

[15] M.H. Kadivar and S.R. Mohebpour, Finite element dynamic analysis of unsymmetric composite laminated beams with shear effect and rotary inertia under the action of moving loads, Finite Elements in Analysis and Design 29 (1998), 259-273.

[16] L. Fryba, Vibration of solids and structures under moving loads, $3^{\text {rd }}$ Edition, Noordhoff International, Groningen, 1999.

[17] Y. Chen, C.A. Tan and L.A. Bergman, Effects of boundary flexibility on the vibration of a continuum with a moving oscillator, Journal of Vibration and Acoustics 124 (2002), 552-560.

[18] S. Mackertich, Dynamic response of a supported beam to oscillatory moving masses, Journal of Vibration and Control 9 (2003), $1083-1091$.

[19] B.J. Olson, S.W. Shaw and G. Stépán, Nonlinear dynamics of vehicle traction, Vehicle System Dynamics 40(6) (2003), 377-399.

[20] M.H. Kargarnovin and D. Younesian, Dynamics of timoshenko beams on pasternak foundation under moving load, Mechanics Research Communications 31 (2004), 713-723. 
[21] P. Lou, G.L. Dai and Q.Y. Zeng, Dynamic analysis of a timoshenko beam subjected to moving concentrated forces using the finite element method, Shock and Vibration 14(6) (2007), 459-468.

[22] P. Lou, Finite-element formulae for calculating the sectional forces of a Bernoulli-Euler beam on continuously viscoelastic foundation subjected to concentrated moving loads, Shock and Vibration 15(2) (2008), 147-162.

[23] M.T. Ahmadian, R.A. Jafari-Talookolaei and E. Esmailzadeh, Dynamics of a laminated composite beam on pasternak-viscoelastic foundation subjected to a moving oscillator, Journal of Vibration and Control 14 (2008), 807-830.

[24] M.T. Ahmadian, R.A. Jafari-Talookolaei and E. Esmailzadeh, Multi-span Laminated composite beam traversed by moving non-rigid masses, 49th AIAA/ASME Structural Dynamics, Schaumburg, IL, USA, April 2008, pp. 7-10.

[25] H. Huang, S. Shen and E. Tutumluer, Moving load on track with asphalt trackbed, Vehicle System Dynamics 48(6) (2010), 737-749.

[26] Y.H. Chen, Y.H. Huang and C.T. Shih, Response of an infinite timoshenko beam on a viscoelastic foundation to a harmonic moving load, Journal of Sound and Vibration 241(5) (2001), 809-824.

[27] M. Zehsaz, M.H. Sadeghi and A. Ziaei Asl, Dynamic response of railway under a moving load, Journal of Applied Sciences 9(8) (2009), 1474-1481.

[28] E.J. Sapountzakis and A.E. Kampitsis, Nonlinear response of shear deformable beams on tensionless nonlinear viscoelastic foundation under moving loads, Journal of Sound and Vibration 330 (2011), 5410-5426.

[29] R.A. Jafari-Talookolaei, M.H. Kargarnovin and M.T. Ahmadian, On the dynamic response of a delaminated composite beam under the motion of an oscillating mass, Journal of Composite Materials, Accepted (In press), DOI: 10.1177/0021998311433344.

[30] R.M. Jones, Mechanics of composite materials, McGraw-Hill, New York, 1975.

[31] S. Krishnaswamy, K. Chandrashekhara and W.Z.B. Wu, Analytical solutions to vibration of generally layered composite beams, Journal of Sound and Vibration 159(1) (1992), 89-99.

[32] S. Timoshenko, Vibration Problems in Engineering, Wiley, New York, 1974. 

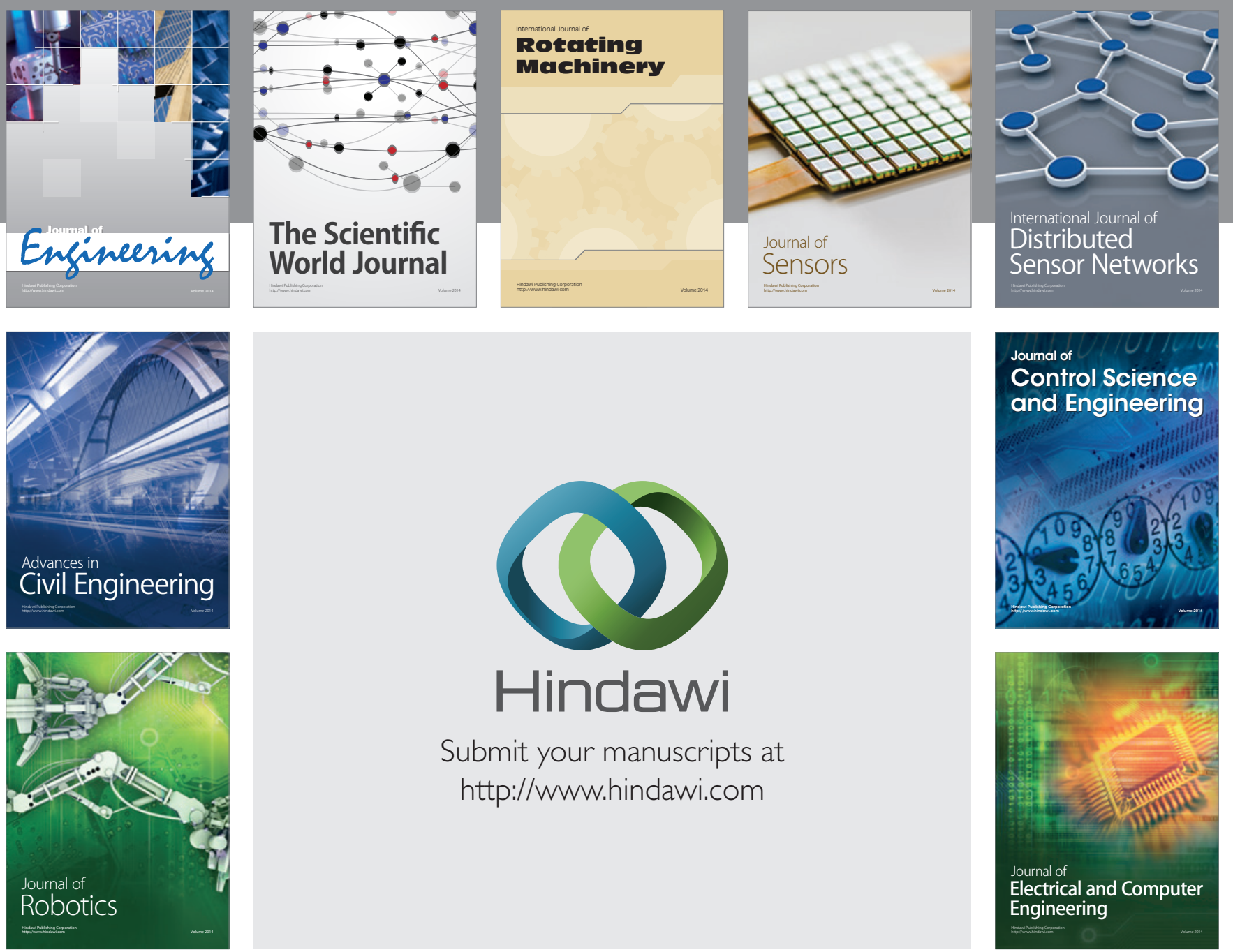

Submit your manuscripts at

http://www.hindawi.com
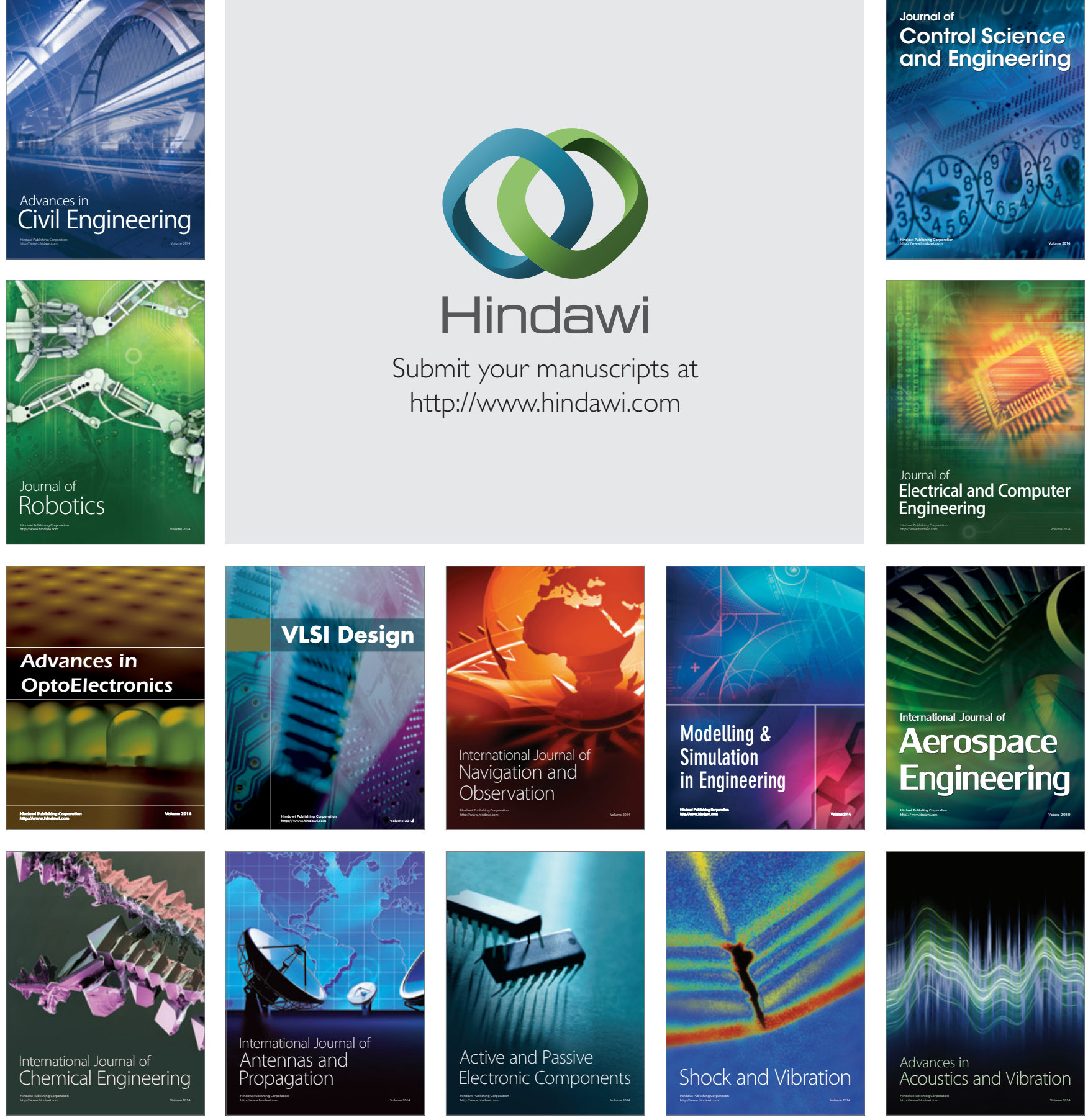